Research Paper

\title{
Integrate analysis of the promote function of Cell division cycle-associated protein family to pancreatic adenocarcinoma
}

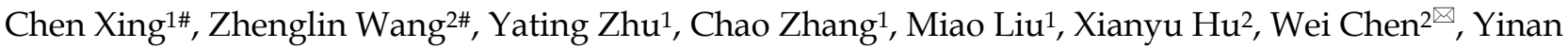 \\ $\mathrm{Du}^{1}{ }^{凶}$ \\ 1. School of Basic Medical Sciences, Anhui Medical University, 230032 Hefei, Anhui, China \\ 2. Department of General Surgery, The First Affiliated Hospital of Anhui Medical University, 230022 Hefei, Anhui, China \\ \# These authors contributed equally for current work. \\ $\bowtie$ Corresponding authors: Yinan Du (duyinannan@126.com), Wei Chen (chenwei366@126.com)
}

() The author(s). This is an open access article distributed under the terms of the Creative Commons Attribution License (https://creativecommons.org/licenses/by/4.0/). See http://ivyspring.com/terms for full terms and conditions.

Received: 2020.09.14; Accepted: 2020.11.19; Published: 2021.01.01

\begin{abstract}
Background: The cell division cycle-associated (CDCA) protein family plays a pivotal role in the regulation of the cell cycle during tumorigenesis and predicts the prognosis of tumors, but an analysis of these proteins in pancreatic adenocarcinoma (PAAD) is still lacking.

Methods: Oncomine and GEPIA were used to observe the expression and prognostic value of eight CDCAs in pan-cancer. Univariate Cox analysis of single CDCAs and multivariate Cox analysis of all eight CDCAs were performed to evaluate the integrated prognostic value of CDCAs, and the results are displayed as hazard ratios (HRs) and $95 \%$ confidence intervals (95\% Cls). K-M plots and receiver operating characteristics curves were used to display the predicted function and accuracy of CDCAs to determine the risk score. Annotation of CDCA-related genes, gene sets enrichment analysis (GSEA) and gene sets variation analysis (GSVA) were performed to reveal the CDCAs that impact biological processes.
\end{abstract}

Results: CDCAs expression in most tumors is higher than that in normal tissues and is associated with a poor prognosis. Regarding PAAD, increased CDCA expression along with advanced PAAD tumor stage, NUF2, CDCA2, CDCA3, CDCA4 and CDCA5 expression are risk factors for poor prognosis, while $C B X 2$ expression is a protective factor $(P<0.05)$. The integrated prognostic value of $C D C A s$ in PAAD patients was validated by SurvExpress in the TCGA-PAAD cohort $(\mathrm{P}<0.001, \mathrm{HR}=2.16,95 \% \mathrm{Cl}$ $=1.41-3.3)$ and the ICGC-PACA cohort $(\mathrm{P}<0.001, \mathrm{HR}=2.56,95 \% \mathrm{Cl}=1.73-3.79)$. Genetic alteration and DNA methylation of CDCAs might not affect the prognosis of PAAD patients. After comparing highand low-risk groups separated by CDCA risk scores, the activated pathways were revealed and included the cell cycle, DNA repair, P53, MYC-targets, E2F-targets and PI3K pathways.

Conclusion: CDCAs can predict the OS prognosis of PAAD patients. The cell cycle, DNA repair, E2F, P53 and PI3K signaling pathways, in which CDCAs are involved, impact the tumorigenesis of PAAD.

\section{Introduction}

Pancreatic cancer is an increasingly common tumor worldwide, and approximately $85 \%$ of cases are consistent with pancreatic adenocarcinoma (PAAD)[1]. PAAD is fourth most frequent cancer and was the seventh most common cause of cancer-related death in 2018 worldwide, accounting for approximately four hundred thirty thousand deaths[2]. Increasing age is a risk factor for the incidence and mortality of PAAD, while PAAD is more frequent in males than in females[2]. Smoking cigarettes, obesity, heavy alcohol abuse and family history are known risk factors for PAAD patients[3-5]. 
Smoking cigarettes can alter the microenvironment of pancreatic tissue, lead to long-standing inflammation, increase onco-miRNA expression, induce KRAS mutations and affect enzyme secretion[6]. Asahina et al. reported that even moderate alcohol use could induce an advanced stage of PAAD in KrasG12D mutant mice[7]. The median overall survival (OS) time for advanced stage PAAD is less than 1 year, while the 5-year survival for patients with all stages of PAAD is less than $10 \%[8,9]$. Therefore, it is necessary to identify prognostic biomarkers for PAAD patients to guide clinical treatment.

An important strategy for tumor therapy focuses on the inhibition of tumor cell proliferation, which is regulated by the three major checkpoints in the cell cycle: G1, G2/M transition and metaphase-toanaphase transition[10]. The cell division cycle-associated (CDCA) protein family is a gene set that is deeply involved in the process of the cell cycle and contains eight homologous proteins: CDCA1 is also known as NUF2 Component of NDC80 Kinetochore Complex (NUF2), and CDCA6 is chromobox 2 (CBX2). CDCA2 can control the protein phosphatase 1 (PP1) $\gamma$-dependent DNA damage response and promotes major mitotic histone $\mathrm{H} 3$ dephosphorylation in a PP1-dependent manner[11, 12]. CDCA3 contains an F-box motif and participates in the G2/M phase of the cell cycle, which can promote cell proliferation through the NF- $\kappa \mathrm{B} /$ cyclin D1 and E2F1/p21 pathways[13, 14]. CDCA5 is recognized as the substrate of the anaphasepromoting complex, which is essential for the stability of cohesion and chromatid binding at the $S$ and G2/M phases and is then degraded in a ubiquitin-dependent manner in the G0/G1 phase[15, 16].

Several studies have illustrated the prognostic value of CDCAs in tumors. Meng et al.[17] reported the increased risk associated with NUF2, CDCA3, CDCA4, CDCA5, CDCA7, and CDCA8 expression in renal cell carcinoma and the decreased risk associated with CDCA2 expression. Zhang et al.[18] found the risk associated with NUF2, CDCA2-5, and CDCA8 expression in endometrial carcinoma, while CDCA7 was a protective factor. However, the prognostic value of CDCAs in PAAD has not been demonstrated. Therefore, in the current study, we elucidated the association between eight CDCAs and the OS of PAAD patients, as well as the integrative CDCA prognostic signature. The potential implications of the genetic alteration and DNA methylation of CDCAs for prognosis were also considered, and the potential signaling pathways impacted by CDCAs were assessed.

\section{Methods}

\section{Pan-cancer analysis for the predicted value of CDCAs}

To globally understand the function of CDCAs, we used the Oncomine publicly online cancer microarray database[19]. This database can display comparable mRNA expression profiles of normal and tumor tissues in different types of cancers obtained from diverse cohorts. The comparation between normal and tumor tissues was analyzed by Student's t-test, with the following thresholds: $\mathrm{P}$ value $<0.05$; fold change $>1.5$; gene rank, top $10 \%$; data type, all. The pan-cancer prognostic values of eight CDCAs were also evaluated by the GEPIA[20]. The hazard ratio (HR) of each gene to the overall survival (OS) in different tumors was calculated by univariate Cox regression analysis.

\section{Prognostic value of CDCAs in PAAD patients}

The comparison of PAAD tumor tissue and normal pancreatic tissue was performed by GEPIA. The mRNA expression levels of eight CDCAs were extracted from 179 PAAD tumor tissues in the TCGA-PAAD cohort and 171 normal pancreatic tissues from the TCGA-PAAD cohort and the GTEx dataset. All the mRNA expression values were pre-normalized by $\log _{2}(\mathrm{TPM}+1)$. The mRNA expression data of eight CDCAs distributed in different tumor stages and grades were downloaded from the ULCAN[21]. The original gene expression files of eight CDCAs and their clinical features were downloaded from the UCSC Xena (https://gdc.xenahubs.net/download/TCGA-PAAD .htseq_fpkm.tsv.gz). The transcripts per million (TPM) read data were calculated from the fragments per kilobase of non-overlapped exons per million fragments (FPKM) value and were then modified to the form of $\log _{2}(\mathrm{TPM}+1)$. A heatmap illustrated by the pheatmap $R$ package was constructed to display the association and distribution between CDCAs and clinical features. K-M survival was used to show the diverse OS outcomes in patients with high or low expression of CDCAs, which are separated by the median value of each gene expression level. The $\mathrm{P}$ value to show the difference in OS outcome in K-M survival was calculated by log-rank test, while the HR and $95 \% \mathrm{CI}$ were obtained by Cox regression analysis in two groups. To investigate the integrative prognostic value of CDCAs, we used a public resource-based survival assessment platform, SurvExpress[22] with the TCGA-PAAD cohort and the ICGC-PACA cohort. The K-M plot and receiver operating characteristic (ROC) curve were displayed with the combined values of the eight CDCAs. 


\section{Genetic alteration and DNA methylation effects on prognosis}

Genetic alterations, including gene mutations and copy number alterations, are the potential factors impacting expression. We evaluated the genetic alterations of CDCAs with Oncoprinter from cBioportal and the impacts of CDCAs on PAAD patient survival[23, 24]. DNA methylation is another risk factor that affects the expression of CDCAs. The influence of DNA methylation on CDCA expression was assessed by DNMIVE[25], and the impact of a single-methylation $\mathrm{CpG}$ site on the OS of PAAD patients was analyzed by MethSurv[26].

\section{CDCAs impact on signaling pathways}

To evaluate the impact of CDCAs on signaling pathways, we first identified the coexpressed genes of eight CDCAs from ULCAN[21]. The thresholds were set as $\mathrm{R}$ higher or equal to 0.3 and $\mathrm{P}$ value less than 0.05 . The genes that met the thresholds for all eight CDCAs were defined as the CDCA-impacted genes (CIGs). Then, we annotated the enrichment of these genes by Metascape[27] to reveal the potential mechanisms regulated by CDCAs. With GSEA analysis of KEGG pathways, we also highlighted the significantly different biological pathways in PAAD patients with CDCAs to determine the high- and low-risk groups[28]. The enrichment score (ES) was summed from the genes from a certain gene set if they met the top genes in all the ranked gene lists and was subtracted if the genes met the bottom genes. Normalized ES (NES) is used to adjust the duplicated analysis among different gene sets. Furthermore, gene-set variation analysis (GSVA) was also employed to assess the activated signaling, which could calculate samplewise gene-set enrichment with a Kolmogorov-Smirnov-like rank statistic; however, genes are often ranked using a kernel estimation of a cumulative density function. We performed GSVA analysis to evaluate the 50 HALLMARK gene signatures.

\section{Statistics}

K-M survival analysis was used to indicate the different OS level of the high and low groups with the 'survminer' package, $\mathrm{R}$ version 3.6.5. Univariate Cox regression analysis was employed to calculate the hazard ratio (HR) and 95\% confidence interval (95\% CI). Comparisons of continuous data between two groups were performed with the Student's T-test. A two-sided $\mathrm{P}$ value less than 0.05 was considered statistically significant.

\section{Results}

\section{CDCAs expression increased in various cancers and was associated with poor prognosis}

We first used Oncomine to globally understand the expression of CDCAs in tumor and normal tissues. We revealed that the eight CDCAs were increased in most tumors, but not leukemia and myeloma (Figure 1A). Furthermore, with the help of GEPIA, we comprehensively evaluated the prognostic value of CDCAs for OS. As shown in Figure 1B, red indicated increased risk, blue indicated decreased risk, and the bold border indicated a $P$ value less than 0.05 . We revealed that the eight CDCAs acted as risk factors for tumorigenesis in most tumors but as protectors in thymoma and thyroid cancer. In PAAD, NUF2, CDCA2, CDCA3, CDCA4 and CDCA5 are risk factors for poor prognosis, while $\mathrm{CBX} 2$ is a protective factor $(\mathrm{P}<0.05)$.

\section{CDCAs were increased in advanced PAAD}

We evaluated the expression levels of eight CDCAs in GEPIA, which contains 179 PAAD tumor tissues from a TCGA-PAAD cohort and 171 normal pancreatic tissues from the TCGA-PAAD cohort and the GTEx database. We found that the expression levels of seven of eight CDCAs were increased in tumor tissues $(P<0.05)$, while there was no significant difference of $\mathrm{CBX} 2$ expression between tumor and normal tissues (Figure 2A). Furthermore, we noted the tendency that the expression of eight CDCAs increased with advancement of the PAAD tumor stage, especially for tumor grade (Figure $2 \mathrm{~B}-\mathrm{C}$ ). The distribution of the expression levels of eight CDCAs and the clinical features of the PAAD patients from the TCGA-PAAD cohort are shown in Figure 2D. We revealed that most CDCAs positively associated with the advanced tumor stage and grade, as well as the outcome of dead (Figure 2E).

\section{CDCAs are associated with the prognosis of PAAD patients}

After combining the mRNA expression data and clinical information, a total of 176 PAAD patients from the TCGA-PAAD cohort were used to analyze the prognostic prediction value of CDCAs. We revealed that higher expression levels of NUF2 $(\mathrm{P}=$ $0.027, \mathrm{HR}=1.6,95 \% \mathrm{CI}=1.055-2.428), \mathrm{CDCA} 2(\mathrm{P}=$ $0.008, \mathrm{HR}=1.76,95 \% \mathrm{CI}=1.156-2.685), \mathrm{CDCA} 3(\mathrm{P}=$ $0.027, \mathrm{HR}=1.6,95 \% \mathrm{CI}=1.056-2.428), \mathrm{CDCA} 4(\mathrm{P}=$ $0.019, \mathrm{HR}=1.65,95 \% \mathrm{CI}=1.086-2.495), \mathrm{CDCA} 5(\mathrm{P}=$ $0.025, \mathrm{HR}=1.61,95 \% \mathrm{CI}=1.061-2.449)$, and CDCA8 ( $\mathrm{P}$ $=0.038, \mathrm{HR}=1.55,95 \% \mathrm{CI}=1.026-2.355)$ indicated an unfavorable OS for patients compared to genes with 
lower expression levels, while higher expression of CBX2 ( $\mathrm{P}=0.013, \mathrm{HR}=0.59,95 \% \mathrm{CI}=0.386-0.892)$ was associated with a favorable prognosis (Figure 3).

\section{Integrated prognostic value of CDCAs in PAAD patients}

The SurvExpress online platform was used to evaluate the integrative prognostic value of the eight CDCAs. For the TCGA-PAAD cohort, the risk score for patients was calculated based on the coefficients of CDCAs, which were generated by multivariate Cox regression analysis. Patients with high risk scores showed poorer OS than those with low risk scores ( $\mathrm{P}$ $<0.001, \mathrm{HR}=2.16,95 \% \mathrm{CI}=1.41-3.3)$. The time-dependent ROC curve showed that the predictive accuracy of CDCAs ranged from 0.662 to
0.878. Increased expression levels of NUF2, CDCA2, CDCA3, CDCA4, CDCA5, CDCA7, and CDCA8 were observed in the high-risk group, while decreased expression of CBX2 was observed in the high-risk group (Figure 4A). We also used the ICGC-PACA cohort to validate the prognostic value of CDCAs. The patients in the high-risk group also had a poor prognosis $(\mathrm{P}<0.001, \mathrm{HR}=2.56,95 \% \mathrm{CI}=1.73-3.79)$. The time-dependent ROC curve revealed that the predictive accuracy of CDCAs ranged from 0.687 to 0.710. Increased expression levels of NUF2, CDCA2, CDCA3, CDCA4, CDCA5, and CDCA8 were observed in the high-risk group of the ICGC-PACA cohort (Figure 4B).
A

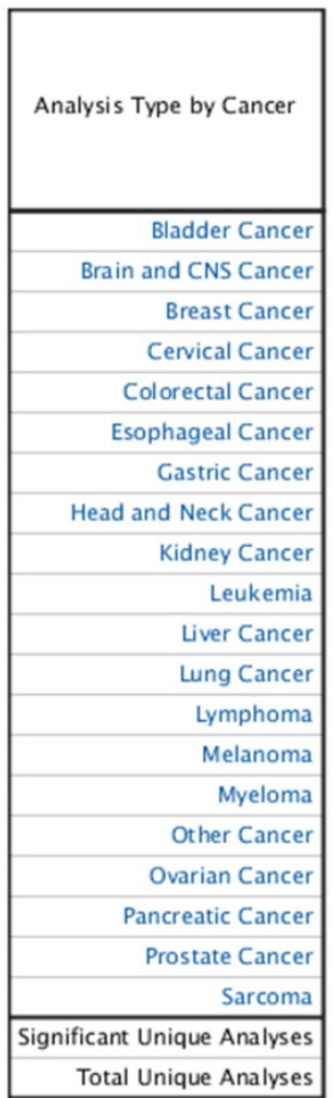

B

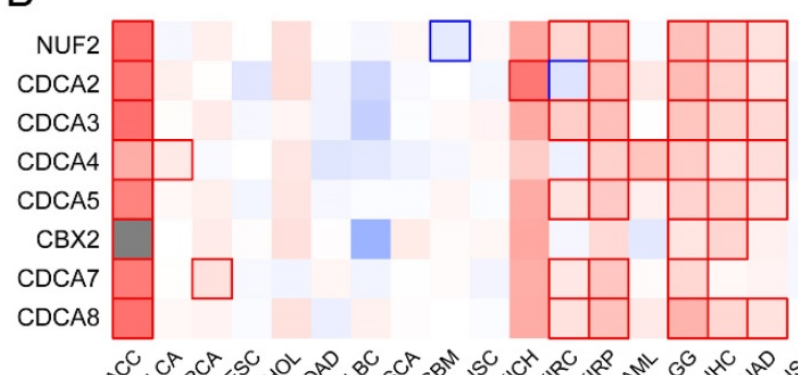

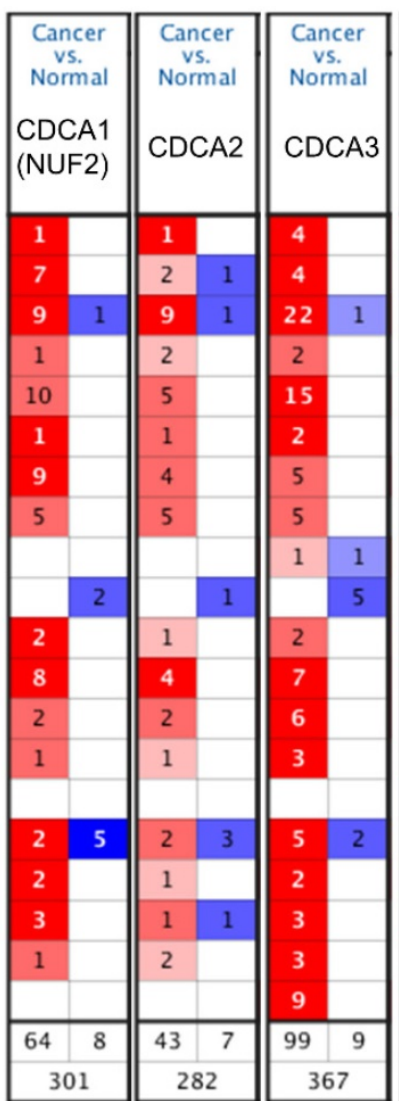

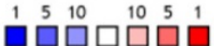
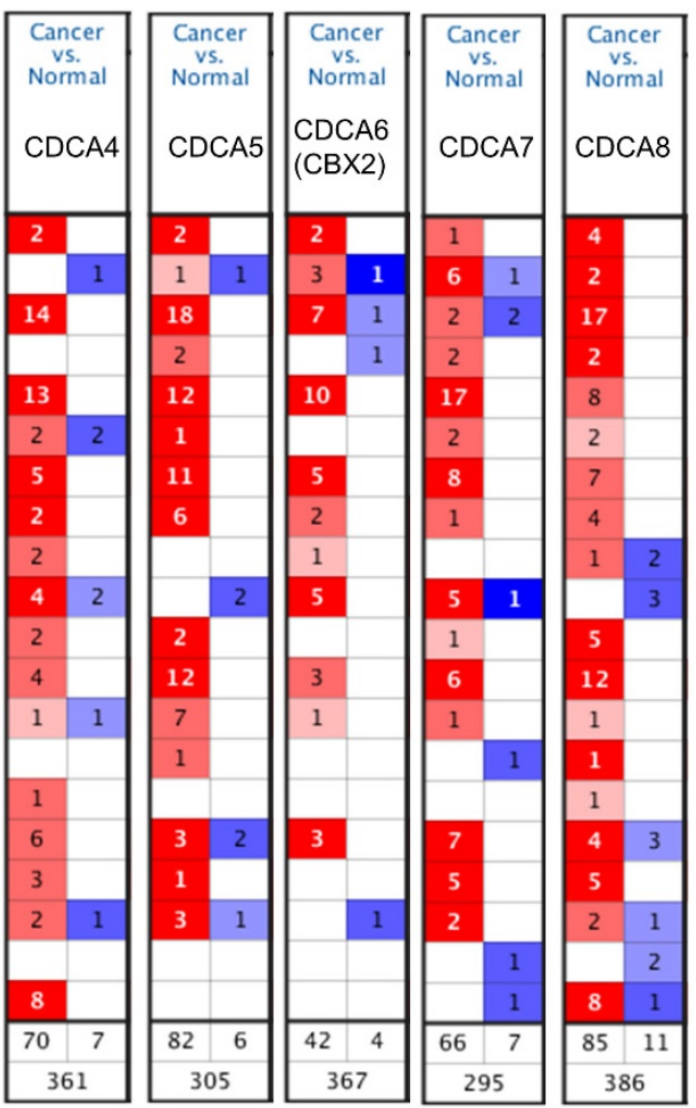

Cell color is determined by the best gene rank percentile for the analyses within the cell.
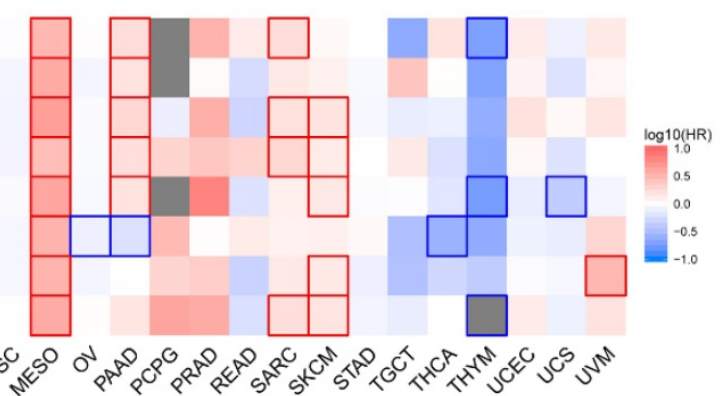

Figure 1. The association between CDCAs with pan-cancer. (A) CDCAs expression in tumor and normal tissues in pan-cancer evaluated by Oncomine; (B) CDCAs correlated with the overall survival of pan-cancer. The red and blue frame indicate the $P$ value less than 0.05 . 

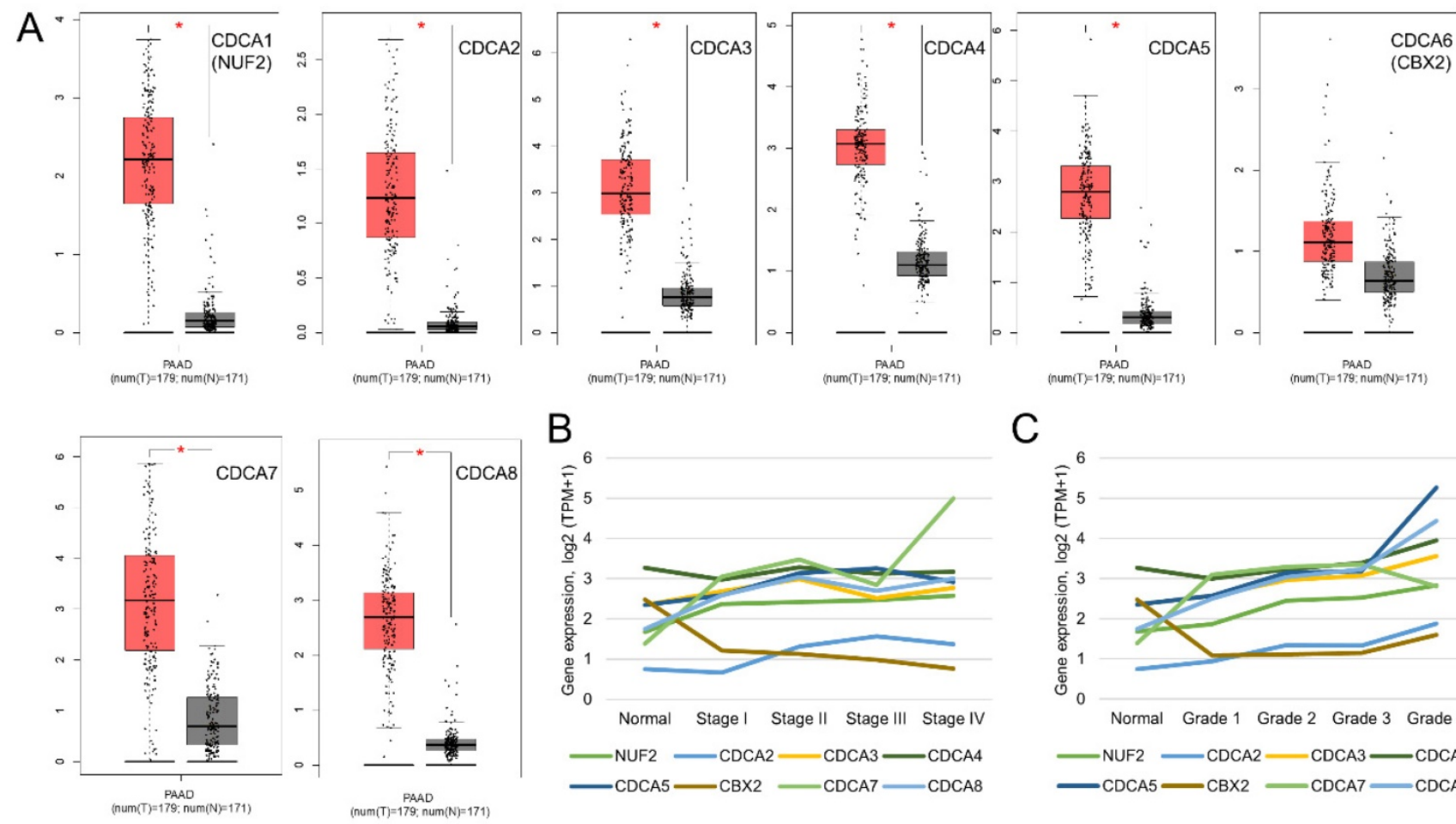

$\mathrm{B}$

C
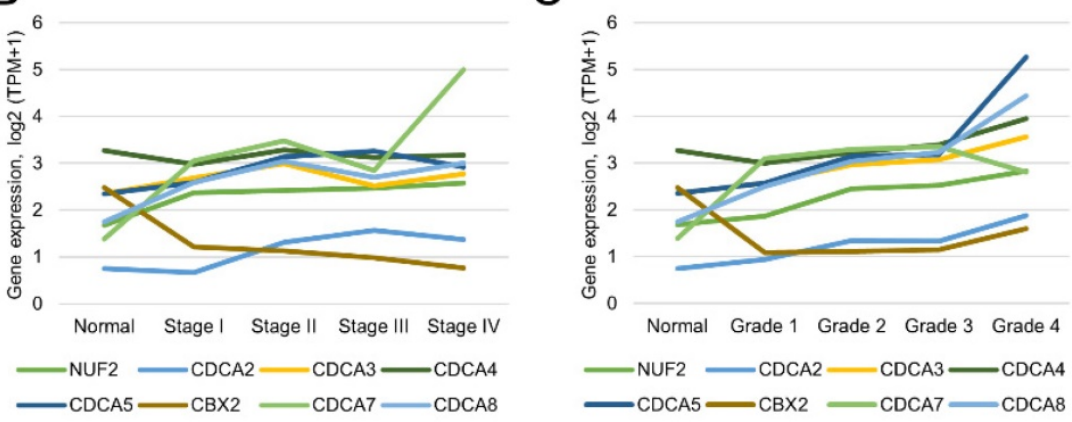

D

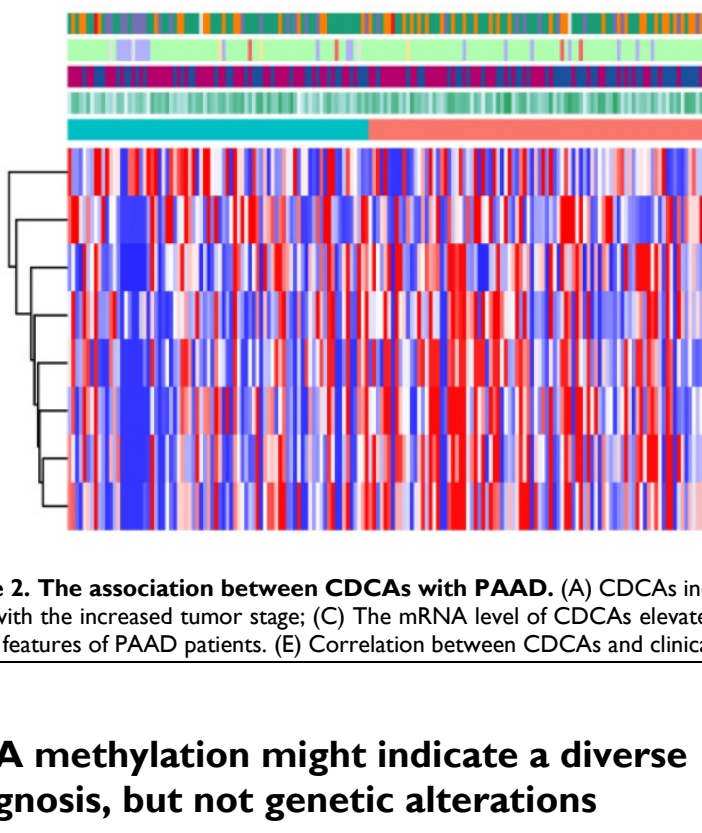

We obtained the genetic alteration data of CDCAs in the TCGA-PAAD cohort from cBioportal. NUF2 had the highest frequency of genetic alteration $(4 \%)$, while only $0.7 \%$ of patients had genetic alterations in CDCA4, CDCA5, and CDCA8 (Figure 5A). Patients with genetic alterations in the CDCAs did not show different OS rates compared with those without the alterations (Figure 5B). Regarding DNA methylation, we first evaluated the promoter methylation and gene expression levels and only found that the promoter methylation of CDCA3 was positively associated with mRNA expression (Figure 5C). Additionally, we revealed the impact of single
CpG to PAAD prognosis, which is displayed in Table 1. In particular, the increased methylation $\beta$ values of the CDCA3-3'UTR-N shelf-cg25700879 site $(P=0.007$, $\mathrm{HR}=1.787)$ and the CDCA3-TSS200/TSS1500island-cg09936970 site $(\mathrm{P}=0.019, \mathrm{HR}=1.622)$ reflected a worse OS (Figure 5D-E).

\section{CDCAs were involved in cell cycle, DNA replication and DNA repair}

To invastigate the mechanism of action of CDCAs, we used different methods. First, we merged the genes with correlations with CDCAs higher or equal to 0.3 based on Pearson analysis and $P$ values less than 0.05 . A total of 445 genes were associated with the eight CDCAs (Figure 6A). The 445 genes were enriched in key biological processes, including 
cell cycle, cell cycle G2/M phase transition, DNA conformation change, DNA replication and DNA repair (Figure 6B-C). Moreover, we compared the activated signaling pathways of the 50 key Hallmark cancer pathways and found that E2F-targets, MYC-targets, P53 pathway, and PI3K signaling were activated in the CDCA-delineated high-risk group and were associated with the DNA repair and G2M checkpoint pathways (Figure 7A). Additionally, similar results were also observed in the GSEA analysis. Activated KEGG cell cycle (Figure 7B), the KEGG P53 signaling pathway (Figure 7C), and DNA repair-associated pathways were observed in the CDCA-delineated high-risk group (Figure 7D, Table 2).
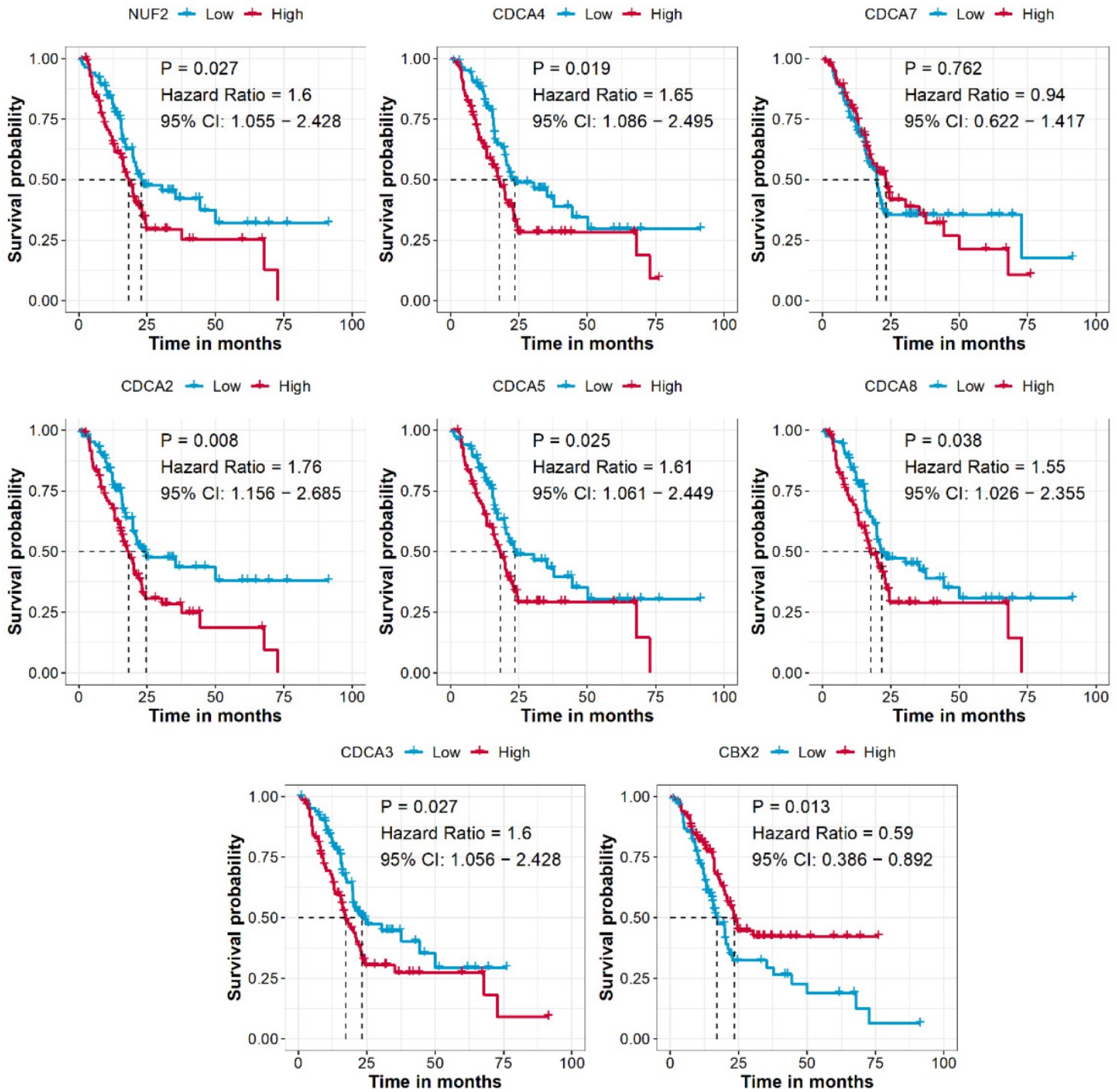

Figure 3. CDCAs associated with the prognosis of PAAD patients. Cut-off value: median expression of CDCAs. 

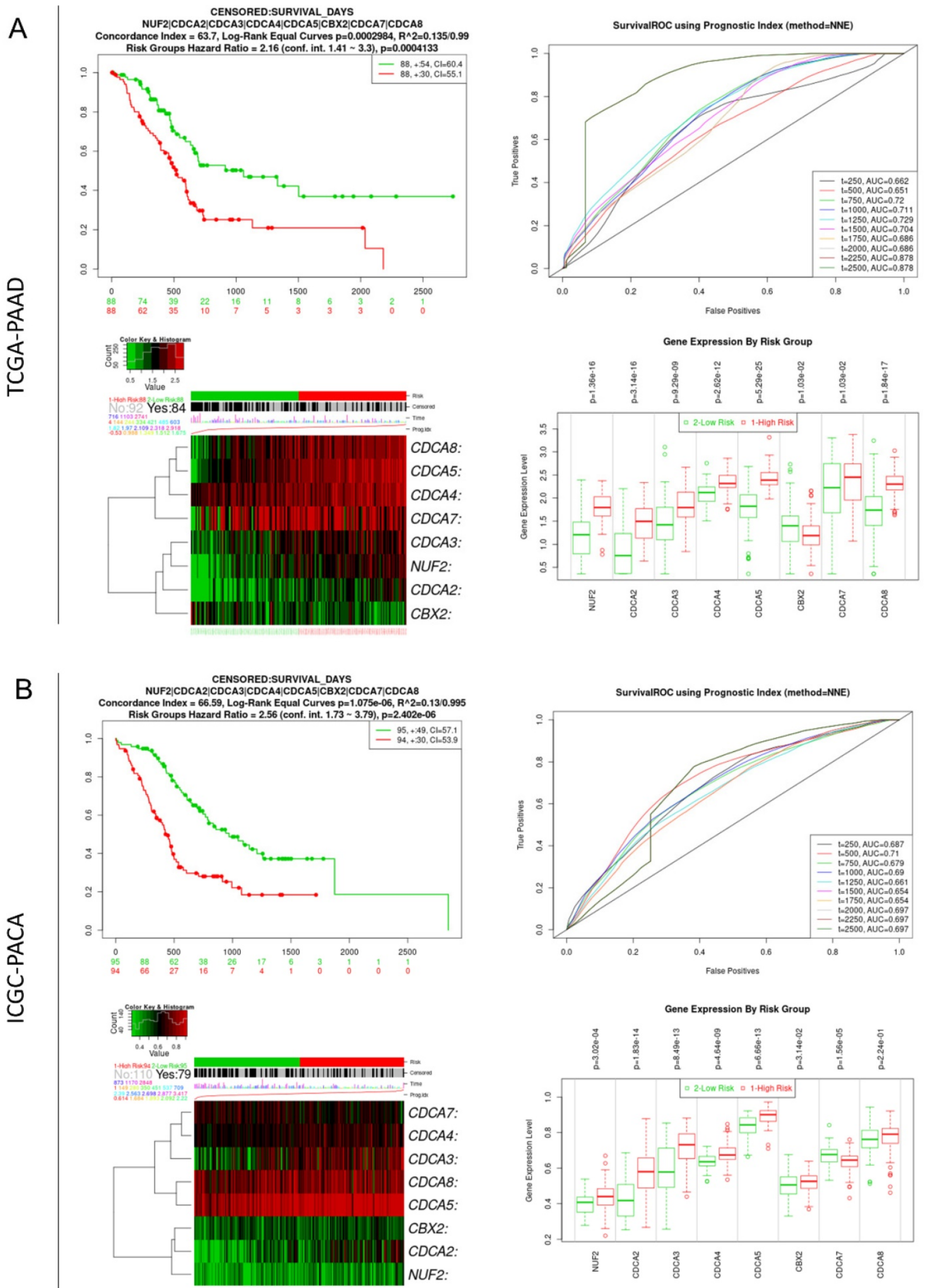

Figure 4. Integrate prognostic value of CDCAs in TCGA-PAAD and ICGC-PACA cohorts. (A) TCGA-PAAD cohort: K-M plot and ROC curve showing the integrate prognostic value of CDCAs, heatmap showing the different distribution of CDCAs expression, bar plot illustrating the mRNA level of CDCAs in high- and low-risk groups; (B) ICGC-PACA cohort: K-M plot and ROC curve showing the integrate prognostic value of CDCAs, heatmap showing the different distribution of CDCAs expression, bar plot illustrating the mRNA level of CDCAs in high- and low-risk groups. 

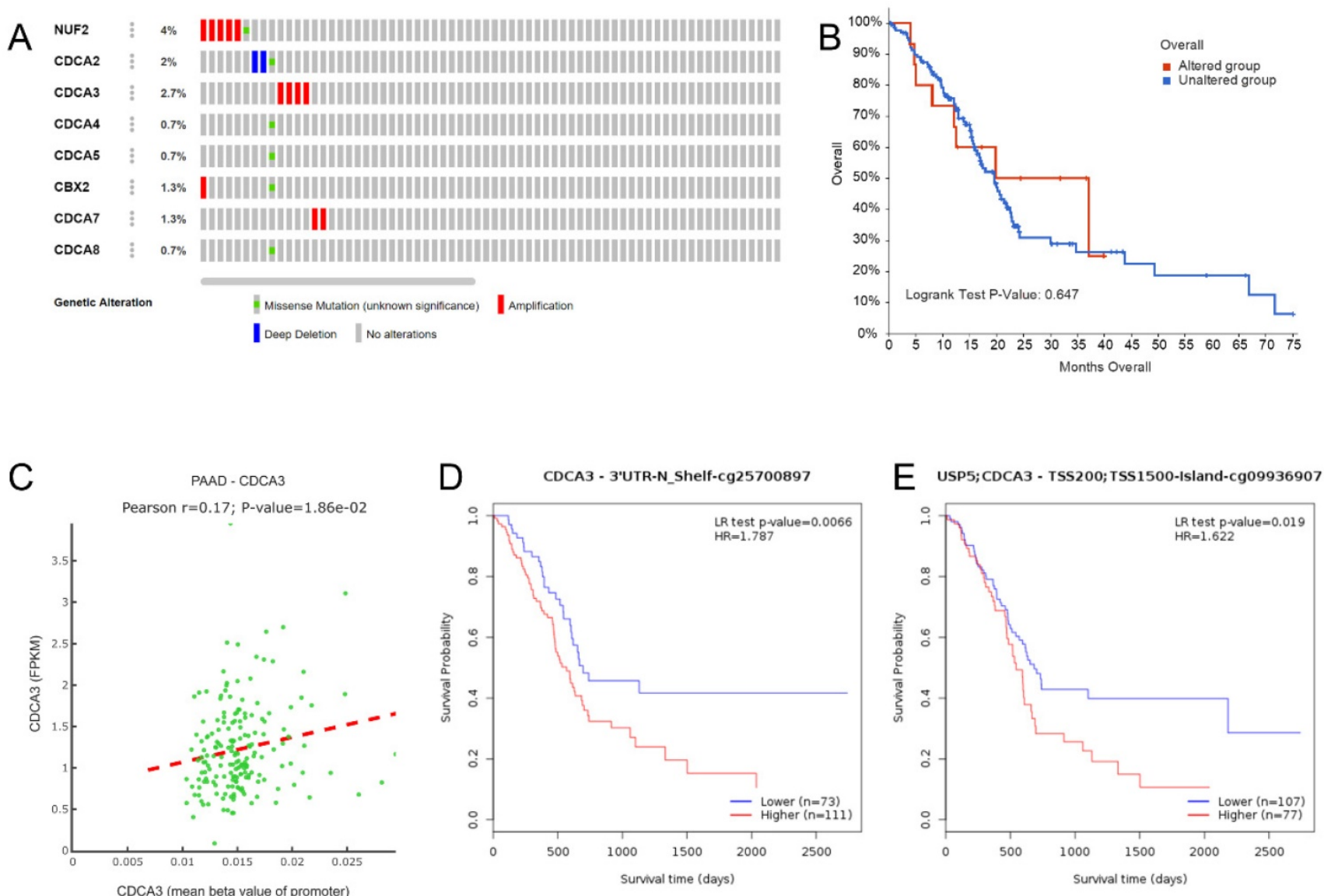

Figure 5. CDCAs genetic alteration and DNA methylation effects to PAAD. (A) Distribution of genetic alteration of eight CDCAs; (B) CDCAs genetic alteration did not impact the OS of PAAD patients; (C) The DNA methylation of CDCA3 impacted to its expression; (D) CDCA3-cg25700897 methylation promoted the poor prognosis of PAAD patients; (E) CDCA3-cg09936970 methylation promoted the poor prognosis of PAAD patients;

Table 1. The prognostic values of CPG sites in the CDCAs by MethSurv.

\begin{tabular}{lllllll}
\hline CpG site & Gene symbol & Group & CpG Island & HR & CI & P value \\
\hline cg21305265 & CDCA2 & TSS1500;1stExon;5'UTR & Island & 0.535 & $0.346-0.828$ & 0.005 \\
cg07446572 & CDCA3 & Body & N_Shore & 0.469 & $0.277-0.795$ & 0.005 \\
cg15942562 & CDCA3 & Body & N_Shore & 0.603 & $0.385-0.945$ & 0.027 \\
cg25700897 & CDCA3 & 3'UTR & N_Shelf & 1.787 & $1.16-2.754$ & 0.009 \\
cg09936907 & CDCA3 & TSS200;TSS1500 & Island & 1.622 & $1.086-2.422$ & 0.018 \\
cg10095089 & CDCA3 & TSS1500;TSS200 & N_Shore & 1.67 & $1.031-2.704$ & 0.037 \\
cg101244440 & CDCA3 & TSS1500;5'UTR & N_Shore & 0.51 & $0.298-0.873$ & 0.014 \\
cg02379052 & CDCA4 & 5'UTR & Island & 0.469 & $0.277-0.794$ & 0.005 \\
cg00047844 & CBX2 & Body & Island & 1.512 & $1.004-2.276$ & 0.048 \\
cg09821032 & CBX2 & TSS1500 & N_Shore & 0.502 & $0.308-0.818$ & 0.006 \\
cg15209885 & CBX2 & Body & S_Shore & 0.537 & $0.314-0.919$ & \\
cg17346145 & CBX2 & Body & N_Shelf & 0.607 & $0.373-0.985$ & 0.023 \\
cg18045515 & CBX2 & TSS1500 & N_Shore & 0.598 & $0.369-0.97$ & 0.043 \\
cg21848700 & CBX2 & TSS1500 & Island & 1.645 & $1.01-2.678$ & 0.037 \\
cg27347140 & CBX2 & TSS1500 & N_Shore & 0.505 & $0.302-0.845$ & 0.045 \\
cg05428978 & CDCA7 & Body & Island & 0.637 & $0.408-0.995$ & 0.009 \\
cg05756320 & CDCA7 & Body & Open_Sea & 1.75 & $1.061-2.888$ & 0.048 \\
cg07302848 & CDCA7 & TSS200 & N_Shore & 0.558 & $0.336-0.927$ & 0.028 \\
c913484295 & CDCA7 & Body & Island & 0.572 & $0.346-0.946$ & 0.024 \\
cg13854747 & CDCA7 & Body & Open_Sea & 1.549 & $1.034-2.32$ & 0.03 \\
cg14906304 & CDCA7 & Body & Open_Sea & 1.661 & $1.005-2.747$ & 0.034 \\
cg17336638 & CDCA7 & Body & Open_Sea & 1.674 & $1.022-2.742$ & 0.048 \\
c921583565 & CDCA7 & TSS1500 & N_Shore & 0.54 & $0.326-0.896$ & 0.041 \\
cg24937696 & CDCA7 & TSS200 & Island & 0.59 & $0.357-0.976$ & 0.017 \\
cg12751733 & CDCA8 & TSS1500 & Island & 1.574 & $1.005-2.466$ & 0.04 \\
cg27171474 & CDCA8 & Body & S_Shelf & 1.58 & $1.027-2.431$ & 0.047 \\
\hline
\end{tabular}




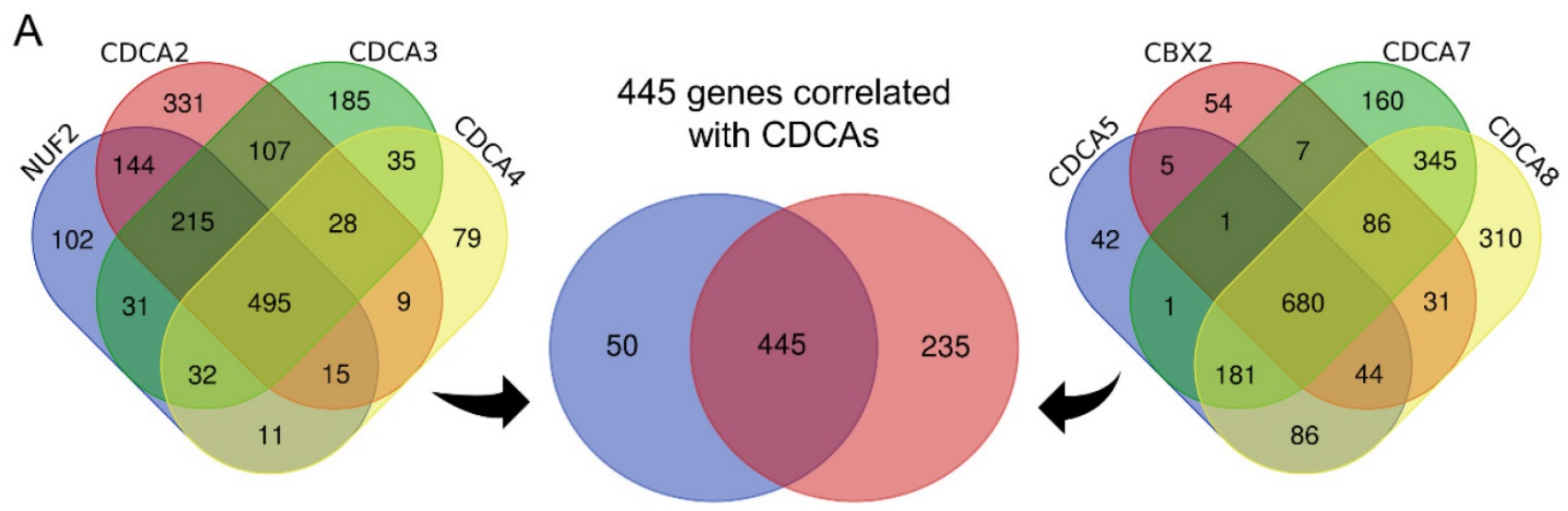

B

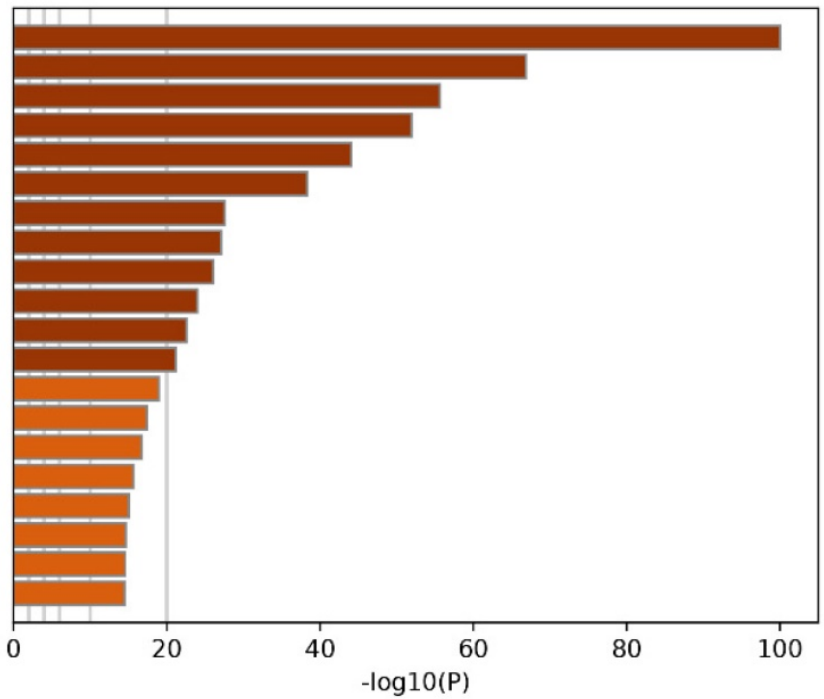

R-HSA-1640170: Cell Cycle GO:0051301: cell division GO:0044772: mitotic cell cycle phase transition GO:0006260: DNA replication GO:0006281: DNA repair GO:0071103: DNA conformation change M129: PID PLK1 PATHWAY GO:0051052: regulation of DNA metabolic process GO:0090068: positive regulation of cell cycle process GO:0044839: cell cycle G2/M phase transition M14: PID AURORA B PATHWAY GO:0051321: meiotic cell cycle M176: PID FOXM1 PATHWAY

R-HSA-3700989: Transcriptional Regulation by TP53 GO:0031570: DNA integrity checkpoint

R-HSA-176974: Unwinding of DNA M40: PID E2F PATHWAY GO:0000910: cytokinesis M1: PID FANCONI PATHWAY R-HSA-8953854: Metabolism of RNA

C

Mitotic cell cycle phase transition

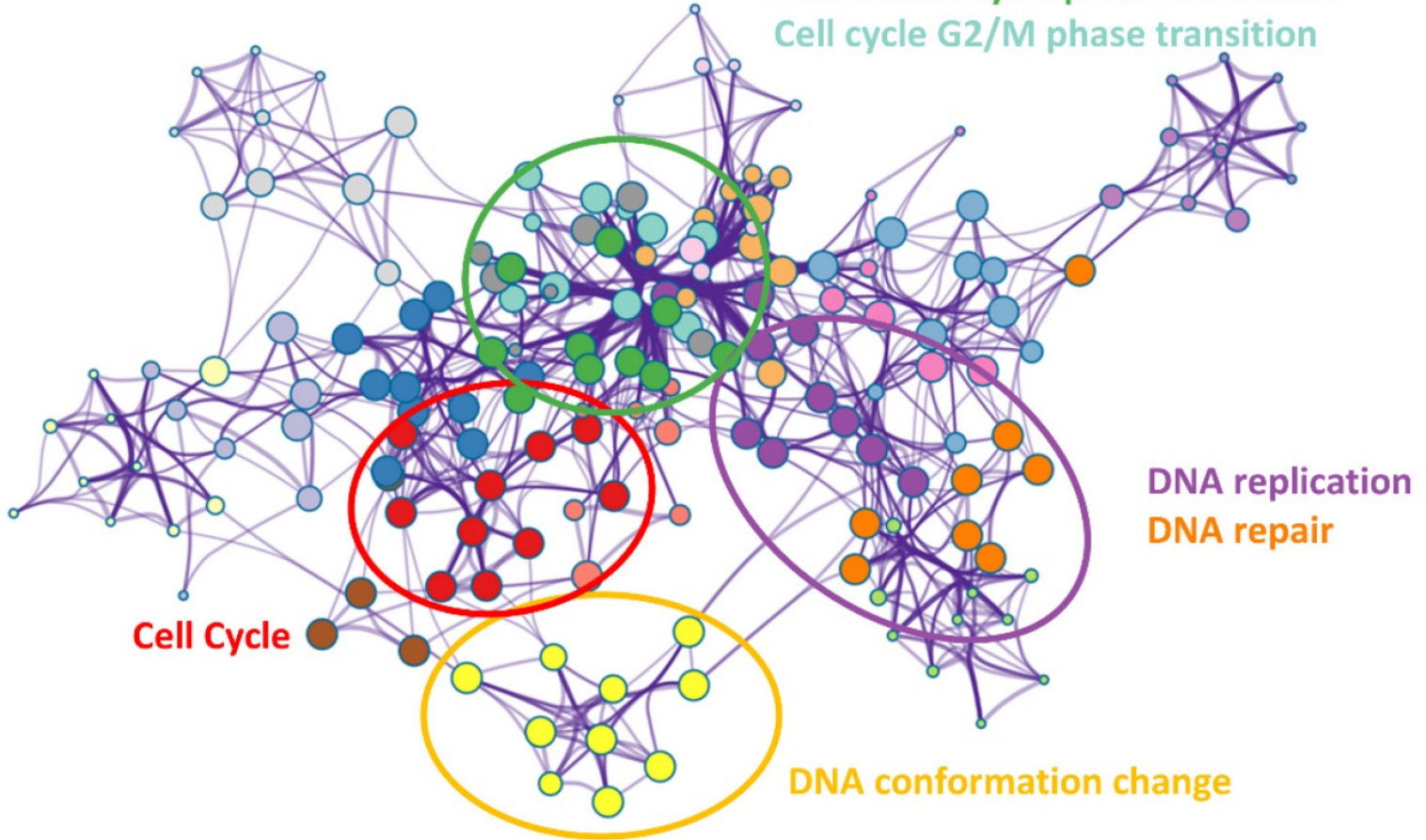

Figure 6. Biological processes implicated by CDCAs (A) The 445 correlated genes to eight CDCAs; (B-C) The annotation of biological processes based on the 445 genes. 

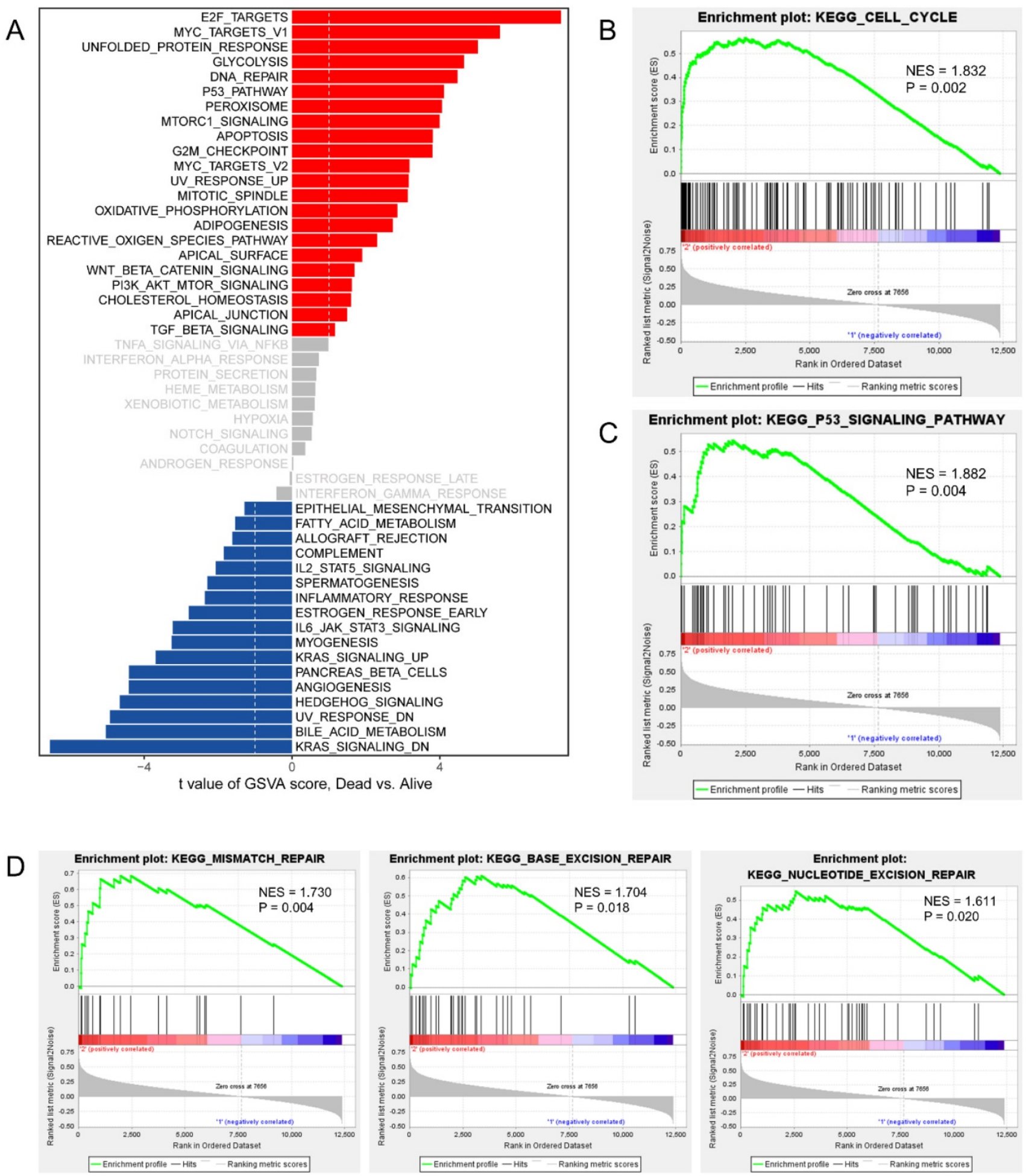

Figure 7. Activated signaling pathways in CDCAs determined high-risk PPAD patients. (A) GSVA analysis showing the activated hallmark pathways; Cell cycle pathway (B), P53 signaling pathway (C) and DNA repair pathways (D) activated in CDCAs determined high-risk PPAD patients.

\section{Discussion}

PAAD is one of the most dangerous tumors and is highly challenging to diagnose in the early stage[29]; thus, most PAADs are well-advanced at the time of diagnosis, while only $7 \%$ of PAADs are at the localized stage at the time of diagnosis[30]. The 5-year survival rates remain as low as $3 \%$ to $15 \%$ [31]. In addition, it is predicted that PAAD will be the second most frequent cause of cancer-related deaths in the United States in 2030[32]. Several factors can increase the risks of PAAD in patients, including pancreatic cystic lesions, familial inherited risk, and type 2 diabetes diagnosed at an age older than 50 years[33, 
34]. In addition, it is important to define the prognostic markers for PAAD to guide clinical treatment. Chung et al.[35] reported the prognostic value of serum fibrinogen to PAAD patients; serum fibrinogen expression was significantly higher in patients with distant metastasis, and the median OS was longer in patients with lower serum fibrinogen levels. Wu et al.[36] identified three immune-related genes (CKLF, ERAP2, and EREG) and determined the prognostic signature of PAAD patients. Patients with high-risk scores were associated with a poor prognosis, with AUC values of 0.612 to 0.687 . Moreover, Suenage et al.[37] used peritoneal lavage tumor DNA (ptDNA) to predict the prognosis of PAAD, as patients with high ptDNA levels have a better disease-free survival and OS.

Table 2. Upregulated gene sets in CDCAs determined high-risk phenotype by GSEA.

\begin{tabular}{lll}
\hline NAME & NES & P value \\
\hline KEGG_PENTOSE_PHOSPHATE_PATHWAY & 1.905 & $<0.001$ \\
KEGG_HOMOLOGOUS_RECOMBINATION & 1.749 & $<0.001$ \\
KEGG_CELL_CYCLE & 1.832 & 0.002 \\
KEGG_OOCYTE_MEIOSIS & 1.765 & 0.004 \\
KEGG_P53_SIGNALING_PATHWAY & 1.882 & 0.004 \\
KEGG_PROTEASOME & 1.826 & 0.004 \\
KEGG_MISMATCH_REPAIR & 1.73 & 0.004 \\
KEGG_PORPHYRIN_AND_CHLOROPHYLL_METABOLISM & 1.797 & 0.006 \\
KEGG_DNA_REPLICATION & 1.791 & 0.006 \\
KEGG_GALACTOSE_METABOLISM & 1.701 & 0.006 \\
KEGG_PROGESTERONE_MEDIATED_OOCYTE_MATURATION N & 1.596 & 0.008 \\
KEGG_PYRIMIDINE_METABOLISM & 1.71 & 0.008 \\
KEGG_THYROID_CANCER & 1.675 & 0.008 \\
KEGG_ONE_CARBON_POOL_BY_FOLATE & 1.681 & 0.008 \\
KEGG_ENDOCYTOSIS & 1.609 & 0.008 \\
KEGG_PATHOGENIC_ESCHERICHIA_COLI_INFECTION & 1.749 & 0.008 \\
KEGG_AXON_GUIDANCE & 1.645 & 0.01 \\
KEGG_AMYOTROPHIC_LATERAL_SCLEROSIS_ALS & 1.639 & 0.01 \\
KEGG_GLYCOLYSIS_GLUCONEOGENESIS & 1.733 & 0.014 \\
KEGG_STARCH_AND_SUCROSE_METABOLISM & 1.63 & 0.015 \\
KEGG_APOPTOSIS & 1.612 & 0.016 \\
KEGG_BASE_EXCISION_REPAIR & 1.704 & 0.018 \\
KEGG_DRUG_METABOLISM_OTHER_ENZYMES & 1.684 & 0.018 \\
KEGG_NUCLEOTIDE_EXCISION_REPAIR & 1.611 & 0.02 \\
KEGG_PURINE_METABOLISM & 1.486 & 0.026 \\
KEGG_PANCREATIC_CANCER & 1.572 & 0.032 \\
KEGG_SPLICEOSOME & 1.57 & 0.034 \\
KEGG_RNA_DEGRADATION & 1.511 & 0.036 \\
KEGG_ADHERENS_JUNCTION & 1.573 & 0.044 \\
KEGG_REGULATION_OF_ACTIN_CYTOSKELETON & 1.503 & 0.046 \\
KEGG_RIG_I_LIKE_RECEPTOR_SIGNALING_PATHWAY & 1.531 & 0.047 \\
\hline
\end{tabular}

In the current study, we tried to illustrate the prognostic value of eight CDCAs in PAAD patients. First, we compared the expression data and found that the eight CDCAs were increased in tumor tissues compared with normal tissues in most cancers and that the CDCAs act as risk factors of tumor OS in most cancers. For PAAD, increased CDCAs were observed in tumor tissues compared to normal tissues and were also observed in the advanced stage and grade PAADs. Increased risk of high CDCA expression was associated with poor prognosis, except for CDCA7 (non-significant) and $\mathrm{CBX} 2$ (opposite result). The prognostic value of CDCAs was also validated through a cell line experiment. Hu et al.[38] found that the increased NUF2 expression in PAAD and determined that NUF2 could alter the proliferation and apoptosis of PAAD cell lines through LncRNA-AF339813. Zou et al. [39] revealed that CDCA3 expression was increased in the PAAD cell lines compared to normal human pancreatic duct epithelial cells, suggesting that knocking down CDCA3 can inhibit cell proliferation and promote cell apoptosis. Wang et al.[40] revealed increased CDCA2 expression in PAAD tumors, and univariate analysis showed that increased CDCA2 expression is a risk factor for PAAD patients. Based on the TCGA-PAAD and ICGC-PACA cohorts, we determined that the integrative risk score based on the expression of eight CDCAs is a good predictor of the prognosis of PAAD patients.

The multicorrelated genes of the eight CDCAs were revealed in a Venn diagram. We found that the CDCAs not only impacted the biological process of the cell cycle but were also involved in DNA replication and repair-associated pathways. With the help of GSVA and GSEA, the impact of CDCAs on the cell cycle and DNA repair pathways was confirmed again, and the E2F, P53, and PI3K signaling pathways were also identified. CDCA2 could recruit the protein phosphatase 1 to chromatin, which impacted the antagonist function of ataxia telangiectasia mutated (ATM)-related signal transduction. DNA damage is fully impacted by the role of ATM kinase; the cascade of ATM kinase phosphorylation can inhibit p53-MDM2 interaction, ultimately leading to p21-induced G1 cell cycle arrest[41]. CDCA4 is a TRIP-Br transcriptional co-factor and can regulate the transcriptional activities of P53 and E2F1 transcription factors and impact the transcriptional regulation and cell fate determination through JUN oncogenes[42]. CBX2 is overexpressed in breast cancer and plays an essential role in tumor progression through the PI3K/AKT pathway[43].

\section{Conclusion}

CDCAs can predict the OS prognosis of PAAD patients. The cell cycle, DNA repair, E2F, P53 and PI3K signaling pathways, in which CDCAs are involved, impact the tumorigenesis of PAAD.

\section{Acknowledgements}

This work was funded by the National Natural Science Foundation of China (No. 31701162)

\section{Availability of data and materials}

The datasets used and/or analyzed during the 
current study are available in TCGA and GEO.

\section{Author Contributions}

Conceived and designed the study: Yinan $\mathrm{Du}$, Wei Chen; Performed the literature search and data extraction: Chen Xing, Zhenglin Wang, Yating Zhu, Chao Zhang, Xianyu Hu; Analyzed the data: Chen Xing, Zhenglin Wang, Miao Liu; Drafted the manuscript: Chen Xing, Zhenglin Wang; All authors approved for the publication.

\section{Competing Interests}

The authors have declared that no competing interest exists.

\section{References}

1. Rawla P, Sunkara T, Gaduputi V. Epidemiology of Pancreatic Cancer: Global Trends, Etiology and Risk Factors. World J Oncol. 2019; 10: 10-27.

2. Bray F, Ferlay J, Soerjomataram I, Siegel RL, Torre LA, Jemal A. Global cancer statistics 2018: GLOBOCAN estimates of incidence and mortality worldwide for 36 cancers in 185 countries. CA Cancer J Clin. 2018; 68: 394-424.

3. Koyanagi YN, Ito H, Matsuo K, Sugawara Y, Hidaka A, Sawada N, et al. Smoking and Pancreatic Cancer Incidence: A Pooled Analysis of 10 Population-Based Cohort Studies in Japan. Cancer Epidemiol Biomarkers Prev. 2019; 28: 1370-8.

4. Lu Y, Gentiluomo M, Lorenzo-Bermejo J, Morelli L, Obazee O, Campa D, et al. Mendelian randomisation study of the effects of known and putative risk factors on pancreatic cancer. J Med Genet. 2020.

5. Lupo F, Piro G, Torroni L, Delfino P, Trovato R, Rusev B, et al. Organoid-Transplant Model Systems to Study the Effects of Obesity on the Pancreatic Carcinogenesis in vivo. Front Cell Dev Biol. 2020; 8: 308.

6. Weissman S, Takakura K, Eibl G, Pandol SJ, Saruta M. The Diverse Involvement of Cigarette Smoking in Pancreatic Cancer Development and Prognosis. Pancreas. 2020; 49: 612-20.

7. Asahina K, Balog S, Hwang E, Moon E, Wan E, Skrypek K, et al. Moderate alcohol intake promotes pancreatic ductal adenocarcinoma development in mice expressing oncogenic Kras. Am J Physiol Gastrointest Liver Physiol. 2020; 318: G265-G76.

8. Ryan DP, Hong TS, Bardeesy N. Pancreatic adenocarcinoma. N Engl J Med. 2014; 371: 2140-1.

9. Vincent A, Herman J, Schulick R, Hruban RH, Goggins M. Pancreatic cancer. Lancet. 2011; 378: 607-20.

10. Kastan MB, Bartek J. Cell-cycle checkpoints and cancer. Nature. 2004; 432: 316-23

11. Thadani R, Uhlmann F, Heeger S. Condensin, chromatin crossbarring and chromosome condensation. Curr Biol. 2012; 22: R1012-21.

12. Qian J, Lesage B, Beullens M, Van Eynde A, Bollen M. PP1/Repo-man dephosphorylates mitotic histone $\mathrm{H} 3$ at $\mathrm{T} 3$ and regulates chromosomal aurora B targeting. Curr Biol. 2011; 21: 766-73.

13. Qian W, Zhang Z, Peng W, Li J, Gu Q, Ji D, et al. CDCA3 mediates p21-dependent proliferation by regulating E2F1 expression in colorectal cancer. Int J Oncol. 2018; 53: 2021-33.

14. Zhang W, Lu Y, Li X, Zhang J, Zheng L, Zhang W, et al. CDCA3 promotes cell proliferation by activating the NF-kappaB/cyclin D1 signaling pathway in colorectal cancer. Biochem Biophys Res Commun. 2018; 500: 196-203.

15. Zhang N, Pati D. Sororin is a master regulator of sister chromatid cohesion and separation. Cell Cycle. 2012; 11: 2073-83.

16. Nishiyama T, Ladurner R, Schmitz J, Kreidl E, Schleiffer A, Bhaskara V, et al. Sororin mediates sister chromatid cohesion by antagonizing Wapl. Cell. 2010; 143: 737-49.

17. Meng J, Gao L, Zhang M, Gao S, Fan S, Liang C. Systematic investigation of the prognostic value of cell division cycle-associated proteins for clear cell renal cell carcinoma patients. Biomark Med. 2020; 14: 223-38.

18. Zhang W, Qiu X, Sun D, Zhang D, Qi Y, Li X, et al. Systematic Analysis of the Clinical Relevance of Cell Division Cycle Associated Family in Endometrial Carcinoma. Journal of Cancer. 2020; 11: 5588-600.

19. Rhodes DR, Yu J, Shanker K, Deshpande N, Varambally R, Ghosh D, et al. ONCOMINE: a cancer microarray database and integrated data-mining platform. Neoplasia. 2004; 6: 1-6.
20. Tang Z, Kang B, Li C, Chen T, Zhang Z. GEPIA2: an enhanced web server for large-scale expression profiling and interactive analysis. Nucleic Acids Res. 2019; 47: W556-W60.

21. Chandrashekar DS, Bashel B, Balasubramanya SAH, Creighton CJ, Ponce-Rodriguez I, Chakravarthi B, et al. UALCAN: A Portal for Facilitating Tumor Subgroup Gene Expression and Survival Analyses. Neoplasia. 2017; 19: 649-58.

22. Aguirre-Gamboa $\mathrm{R}$, Gomez-Rueda $\mathrm{H}$, Martinez-Ledesma $\mathrm{E}$ Martinez-Torteya A, Chacolla-Huaringa R, Rodriguez-Barrientos A, et al. SurvExpress: an online biomarker validation tool and database for cancer gene expression data using survival analysis. PLoS One. 2013; 8: e74250.

23. Gao J, Aksoy BA, Dogrusoz U, Dresdner G, Gross B, Sumer SO, et al. Integrative analysis of complex cancer genomics and clinical profiles using the cBioPortal. Sci Signal. 2013; 6: pl1.

24. Cerami E, Gao J, Dogrusoz U, Gross BE, Sumer SO, Aksoy BA, et al. The cBio cancer genomics portal: an open platform for exploring multidimensional cancer genomics data. Cancer Discov. 2012; 2: 401-4.

25. Ding $W$, Chen $G$, Shi T. Integrative analysis identifies potential DNA methylation biomarkers for pan-cancer diagnosis and prognosis. Epigenetics. 2019; 14: 67-80.

26. Modhukur V, Iljasenko T, Metsalu T, Lokk K, Laisk-Podar T, Vilo J. MethSurv: a web tool to perform multivariable survival analysis using DNA methylation data. Epigenomics. 2018; 10: 277-88.

27. Zhou Y, Zhou B, Pache L, Chang M, Khodabakhshi AH, Tanaseichuk O, et al. Metascape provides a biologist-oriented resource for the analysis of systems-level datasets. Nat Commun. 2019; 10: 1523.

28. Subramanian A, Tamayo P, Mootha VK, Mukherjee S, Ebert BL, Gillette MA, et al. Gene set enrichment analysis: a knowledge-based approach for interpreting genome-wide expression profiles. Proc Natl Acad Sci U S A. $2005 ; 102: 15545-50$

29. Pereira SP, Oldfield L, Ney A, Hart PA, Keane MG, Pandol SJ, et al. Early detection of pancreatic cancer. Lancet Gastroenterol Hepatol. 2020; 5: 698-710.

30. Jemal A, Siegel R, Ward E, Hao Y, Xu J, Thun MJ. Cancer statistics, 2009. CA Cancer J Clin. 2009; 59: 225-49.

31. Arnold M, Rutherford MJ, Bardot A, Ferlay J, Andersson TM, Myklebust TA, et al. Progress in cancer survival, mortality, and incidence in seven high-income countries 1995-2014 (ICBP SURVMARK-2): a population-based study. Lancet Oncol. 2019; 20: 1493-505.

32. Rahib L, Smith BD, Aizenberg R, Rosenzweig AB, Fleshman JM, Matrisian LM. Projecting cancer incidence and deaths to 2030: the unexpected burden of thyroid, liver, and pancreas cancers in the United States. Cancer Res. 2014; 74: 2913-21.

33. Sharma A, Smyrk TC, Levy MJ, Topazian MA, Chari ST. Fasting Blood Glucose Levels Provide Estimate of Duration and Progression of Pancreatic Cancer Before Diagnosis. Gastroenterology. 2018; 155: 490-500 e2.

34. Vege SS, Ziring B, Jain R, Moayyedi P, Clinical Guidelines C, American Gastroenterology A. American gastroenterological association institute guideline on the diagnosis and management of asymptomatic neoplastic pancreatic cysts. Gastroenterology. 2015; 148: 819-22; quize12-3.

35. Chung KH, Lee JC, Lee J, Cho IK, Kim J, Jang W, et al. Serum fibrinogen as a diagnostic and prognostic biomarker for pancreatic ductal adenocarcinoma. Pancreatology. 2020.

36. Wu G, Deng Z, Jin Z, Wang J, Xu B, Zeng J, et al. Identification of Prognostic Immune-Related Genes in Pancreatic Adenocarcinoma and Establishment of a Prognostic Nomogram: A Bioinformatic Study. Biomed Res Int. 2020; 2020: 1346045.

37. Suenaga M, Fujii T, Yamada S, Hayashi M, Shinjo K, Takami H, et al. Peritoneal Lavage Tumor DNA as a Novel Biomarker for Predicting Peritoneal Recurrence in Pancreatic Ductal Adenocarcinoma. Ann Surg Oncol. 2020.

38. Hu P, Shangguan J, Zhang L. Downregulation of NUF2 inhibits tumor growth and induces apoptosis by regulating lncRNA AF339813. Int J Clin Exp Pathol. 2015; 8: 2638-48.

39. Zou RC, Guo ZT, Wei D, Shi ZT, Ye ZC, Zhai G, et al. Downregulation of CDCA3 expression inhibits tumor formation in pancreatic cancer. Neoplasma. 2020

40. Wang MY, Niu ZY, Gao XG, Zhou L, Liao Q, Zhao YP. Prognostic Impact of Cell Division Cycle Associated 2 Expression on Pancreatic Ductal Adenocarcinoma. Chin Med Sci J. 2016; 31: 149-54.

41. Uchida F, Uzawa K, Kasamatsu A, Takatori H, Sakamoto Y, Ogawara K, et al. Overexpression of CDCA2 in human squamous cell carcinoma: correlation with prevention of G1 phase arrest and apoptosis. PLoS One. 2013; 8: e56381

42. Tategu M, Nakagawa H, Hayashi R, Yoshida K. Transcriptional co-factor CDCA4 participates in the regulation of JUN oncogene expression. Biochimie. 2008; 90: 1515-22. 
43. Zheng S, Lv P, Su J, Miao K, Xu H, Li M. Overexpression of CBX2 in breast cancer promotes tumor progression through the PI3K/AKT signaling pathway. Am J Transl Res. 2019; 11: 1668-82. 